\title{
Dietary changes during pregnancy and the postpartum period in Singaporean Chinese, Malay and Indian women: the GUSTO birth cohort study
}

\author{
Ling-Wei Chen ${ }^{1}$, Yen Ling Low ${ }^{1}$, Doris Fok ${ }^{2}$, Wee Meng Han ${ }^{3}$, Yap Seng Chong ${ }^{4}$, \\ Peter Gluckman ${ }^{5}$, Keith Godfrey ${ }^{6}$, Kenneth Kwek ${ }^{3}$, Seang-Mei Saw ${ }^{1}$, Shu E Soh ${ }^{1,7}$, \\ Kok Hian Tan ${ }^{3}$, Mary Foong Fong Chong ${ }^{8, *}$ and Rob M van Dam ${ }^{1,9, *}$ \\ ${ }^{1}$ Saw Swee Hock School of Public Health, National University of Singapore, Block MD3, 16 Medical Drive, \\ Singapore 117597: ${ }^{2}$ National University Health System, Singapore: ${ }^{3}$ KK Women's and Children's Hospital, \\ Singapore: ${ }^{4}$ Department of Obstetrics and Gynaecology, Yong Loo Lin School of Medicine, National University \\ of Singapore, Singapore: ${ }^{5}$ Growth, Development and Metabolism Program, Singapore Institute for Clinical \\ Sciences, A*STAR, Singapore: ${ }^{6}$ Medical Research Council Lifecourse Epidemiology Unit and NIHR Southampton \\ Biomedical Research Centre, University of Southampton and University Hospital Southampton NHS Foundation \\ Trust, Southampton, UK: ${ }^{7}$ Department of Paediatrics, Yong Loo Lin School of Medicine, National University of \\ Singapore, Singapore: ${ }^{8} \mathrm{Clinical}$ Nutrition Research Centre, Singapore Institute for Clinical Sciences, 14 Medical \\ Drive, \#07-02, Singapore 1 17599: ${ }^{9}$ Department of Medicine, Yong Loo Lin School of Medicine, National \\ University of Singapore, Singapore
}

Submitted 14 August 2012: Final revision received 16 May 2013: Accepted 29 May 2013: First published online 28 June 2013

\begin{abstract}
Objective: To examine changes in food consumption during pregnancy and the postpartum period in women of major Asian ethnic groups.

Design: Using interviewer-administered questionnaires, we assessed changes in food consumption during pregnancy (26-28 weeks' gestation) and the postpartum period (3 weeks after delivery) as compared with the usual pre-pregnancy diet.

Setting: Singapore.

Subjects: Pregnant women ( $n$ 1027) of Chinese, Malay and Indian ethnicity (mean age $30 \cdot 4$ (SD $5 \cdot 2$ ) years) who participated in the Growing Up in Singapore Towards healthy Outcomes (GUSTO) study.

Results: During pregnancy, participants tended to increase their consumption of milk, fruit and vegetables and decrease their consumption of tea, coffee, soft drinks and seafood (all $P<0 \cdot 001$ ). Most participants reported adherence to traditional restrictions ('confinement') during the early postpartum period (Chinese: $94 \cdot 8 \%$, Malay: $91 \cdot 6 \%$, Indian: $79 \cdot 6 \%$ ). During the postpartum period, participants tended to increase their consumption of fish and milk-based drinks and decrease their consumption of noodles, seafood, and chocolates and sweets (all $P<0 \cdot 001$ ). Ethnic differences in food consumption were pronounced during the postpartum period. For example, most Chinese participants $(87 \cdot 2 \%)$ increased their ginger consumption during the postpartum period as compared with smaller percentages of Malays $(31 \cdot 8 \%)$ and Indians $(40 \cdot 8 \% ; P$ for ethnic difference $<0 \cdot 001)$. Similar ethnic differences were observed for cooking wine/alcohol, herbs and spices, and herbal tea consumption.

Conclusions: Marked changes in food consumption that reflect both modern dietary recommendations and the persistence of traditional beliefs were observed in Singaporean women during pregnancy and the postpartum period. Traditional beliefs should be considered in interventions to improve dietary intakes during these periods.
\end{abstract}

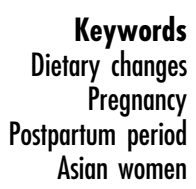

Multiple lines of evidence indicate that maternal diet during pregnancy and the postpartum period has important effects on the offspring's health ${ }^{(1,2)}$. It is widely recognized that poor maternal nutritional status is associated with adverse birth outcomes ${ }^{(1)}$. Moreover, the diet of a lactating mother influences the composition of breast $\mathrm{milk}^{(2)}$. In Asia, the dietary choices of women during pregnancy and the postpartum period may be heavily influenced by traditional medical theories and cultural beliefs surrounding these periods ${ }^{(3-5)}$. In general, pregnancy is considered 
to be a 'hot' state, but with blood loss after giving birth the woman moves into a state of excess 'cold ${ }^{(5,6)}$. It is believed that a 'hot-cold balance' should be maintained in order to stay healthy ${ }^{(5,7,8)}$ and that this can be achieved by following special prescriptive diets during pregnancy and the initial postpartum period, commonly termed 'the confinement period'.

The confinement period is the period immediately after delivery when women are confined to their homes and are expected to observe a broad set of restrictive prescriptions and proscriptions regarding diet and other behaviours ${ }^{(4)}$. For instance, Chinese women are prohibited to take baths and are advised to stay in bed as much as possible during the confinement period ${ }^{(4)}$. The confinement period is practised by major Asian ethnic groups in Asia, including Chinese, Malays and Indians in their countries of origin and by migrants of these ethnic groups living in other parts of the world such as the USA ${ }^{(9)}$, Scotland ${ }^{(10)}$, Australia ${ }^{(11)}$, Taiwan ${ }^{(12)}$ and Singapore ${ }^{(13)}$. The confinement period is usually practised for $30-45 \mathrm{~d}^{(7)}$.

Studies in the literature have focused on confinement practices and behaviours but data on the dietary changes in pregnant and postpartum women during confinement are sparse. An understanding of dietary changes during pregnancy and the postpartum period in these Asian groups could allude to the extent of influence traditional beliefs have on their diets. This could aid the development of culturally sensitive antenatal and postpartum dietary interventions necessary to improve maternal nutrition for the health benefits of the mother and child.

Singapore is a multi-ethnic South-East Asian country that includes Chinese, Malay and Indian populations. We assessed the dietary changes of Chinese, Malay and Indian women in Singapore during pregnancy and the confinement period.

\section{Methods}

We used data from the Growing Up in Singapore Towards healthy Outcomes (GUSTO) study. The GUSTO study is a birth cohort study designed to investigate the effect of early-life events on the risk of developing metabolic diseases later in life. The study involves detailed assessments of pregnant women and their children after birth. The study was granted ethical approval by the Institutional Review Board of the KK Women's and Children's Hospital (KKH) and National University Hospital (NUH). Written informed consent was collected from all participants upon recruitment.

\section{Study population}

Pregnant women who were attending their antenatal care ( $<14$ weeks' gestation) in KKH and NUH, which house the major public maternity units in Singapore, were recruited into the GUSTO study from June 2009 to
September 2010. The inclusion criteria included age range between 18 and 50 years, intention to finally deliver in $\mathrm{KKH}$ and NUH, intention to reside in Singapore for the next 5 years, and willingness to donate cord, cord blood and placenta. Only Chinese, Malay and Indian women whose parents and whose husband's parents were of the same ethnicity were included in the study. Women with significant health conditions such as type 1 diabetes mellitus and psychosis were excluded.

Only GUSTO participants who completed the questionnaires on changes in food consumption during pregnancy and the postpartum period were included in the present dietary study. After excluding those who provided incomplete nutritional information ( $n$ 136; for details, see Supplementary Figure 1 in the online supplementary material), the final numbers of participants for the present dietary study were 1019 for diet during pregnancy and 895 for diet during the postpartum period.

\section{Assessment of covariates}

Data on ethnicity, age, education level and monthly household income were assessed during the recruitment of pregnant women attending their antenatal care. To calculate pre-pregnancy body mass indices of the participants, self-reported pre-pregnancy weights of the participants were obtained during the recruitment while heights of the participants were measured during the pregnancy clinic visit, when women were in the 26th to 28th week of pregnancy. Weights were also measured during this clinic visit. Standing heights were measured with a stadiometer (model 213; Seca, Hamburg, Germany) whereas weights were measured using digital scales (model 803; Seca). Both measurements were taken separately two times with bare feet. If the first two measurements differed by $\geq 1 \mathrm{~cm}$ or $\geq 200 \mathrm{~g}$ for height or weight, a third measurement was taken for the average calculation. Weight gains up to 26-28 weeks' gestation were calculated by subtracting selfreported pre-pregnancy weights from weights measured at 26-28 weeks' gestation. Information about cigarette smoking and alcohol consumption habits before and during pregnancy was also gathered during the pregnancy clinic visit at 26-28 weeks' gestation. A $24 \mathrm{~h}$ recall was administered during the same visit. From the $24 \mathrm{~h}$ recall, daily energy and major nutrient intakes were calculated. Breast-feeding and postpartum practices (whether the participants went through confinement and how so if they did) were assessed during the 3-week postpartum home visit. All questionnaires were administered by trained interviewers.

\section{Assessment of dietary changes}

The questions used to assess dietary changes during pregnancy were part of the interviewer-administered questionnaire used at the pregnancy clinic visit, while those on dietary changes during the postpartum period were included in the interviewer-administered questionnaire used at the 3-week postpartum home visit. Specifically, 
participants were asked to rate whether they consumed 'more', 'less', 'same as before' or 'do not usually eat' for a list of foods during pregnancy and the postpartum period, as compared with their usual pre-pregnancy diets. For clarity in describing dietary changes, we subsequently refer to the 'more' category as 'increased' and to the 'less' category as 'decreased'. The food lists contained seventeen items for the pregnancy questionnaire and thirty-two items for the postpartum questionnaire. The food items represent major food groups and different food categories that can provide useful information on dietary changes during these periods.

To obtain more insight into the accuracy of our questionnaire on dietary changes, we compared the mean intakes of selected nutrients (as recorded in $24 \mathrm{~h}$ recall) for the 'increased' and 'decreased' groups as defined by the dietary changes questionnaire. For this purpose, we used information on three nutrients for which foods of interest are a major source (i.e. Ca for milk, fibre and vitamin C for fruit and vegetables). We found that the mean Ca intake of participants who increased their intakes of milk was 233.6 (95\% CI 167.2, 300.1) $\mathrm{mg} / \mathrm{d}$ higher as compared with participants who decreased their milk intakes. Participants who increased their intakes of vegetables had a $4 \cdot 2(95 \%$ CI $2 \cdot 3,6 \cdot 2) \mathrm{g} / \mathrm{d}$ higher mean dietary fibre intake and a $45 \cdot 2(95 \%$ CI $22 \cdot 3,68 \cdot 0) \mathrm{mg} / \mathrm{d}$ higher mean vitamin $\mathrm{C}$ intake as compared with those who decreased their intakes of vegetables. Similarly, the mean intake of dietary fibre was $5 \cdot 3(95 \% \mathrm{CI} 3 \cdot 7,7 \cdot 0) \mathrm{g} / \mathrm{d}$ and the mean intake of vitamin C was $42 \cdot 8(95 \%$ CI $24 \cdot 2,61 \cdot 4) \mathrm{mg} / \mathrm{d}$ higher for participants who reported increased intakes of fruit as compared with participants who reported a decreased intake of fruit. These results suggest that the dietary changes questionnaire captured important changes in dietary intakes.

\section{Statistical analysis}

Sociodemographic information was summarized for the study population and according to ethnicity. One-way ANOVA, the independent-samples $t$ test and Pearson's $\chi^{2}$ test of independence were used to assess differences in sociodemographic characteristics across ethnic groups and between participants who did or did not go through confinement. Post hoc tests (all possible pairwise comparisons) were conducted if the overall tests were significant.

The percentages of the four categories - 'increased', 'decreased', 'same as before' and 'do not usually eat' were summarized for all food items according to ethnicity. For each food item, Pearson's $\chi^{2}$ test of goodness-of-fit was used to test whether the percentages in the 'increased' and 'decreased' categories varied significantly from the expected proportion of $50 \%$ in each category. Pearson's $\chi^{2}$ test of independence was used to assess if there were significant ethnic differences in changes in food consumption. For these tests, only the 'increased' and 'decreased' categories were considered as they reflected dietary changes.
We also conducted several sensitivity analyses. For both pregnancy and the postpartum period, we conducted analyses stratified for pre-pregnancy BMI $\left(<25 \cdot 0 \mathrm{~kg} / \mathrm{m}^{2}\right.$ or $\geq 25 \cdot 0 \mathrm{~kg} / \mathrm{m}^{2}$ ) and weight gain up to $26-28$ weeks' gestation (weight gain less than the mean value or weight gain greater than or equal to the mean value) to examine if results differed substantially. For the postpartum period only, we stratified by breast-feeding practice at 3 weeks postpartum (yes/no). Furthermore, we restricted the analysis to participants who reported going through confinement. In these sensitivity analyses, a $\geq 10 \%$ difference in the percentage of participants in the 'increased' and 'decreased' categories was considered substantial.

All tests were two-sided. $P$ values smaller than $0 \cdot 001$ were considered to be statistically significant to account for the number of tests that were performed (there were forty-nine food items in total). Post hoc tests were conducted with Bonferroni adjustment. All statistical analyses were performed using the statistical software package STATA $10 \cdot 0$.

\section{Results}

\section{Sociodemographic characteristics}

Table 1 shows the characteristics of study participants. The mean age of the study population was $30 \cdot 4$ (SD 5.2) years. The ethnic background of the study population was $56 \cdot 5 \%$ Chinese, $25 \cdot 5 \%$ Malay and $18 \cdot 0 \%$ Indian. More than half of the participants received at least a pre-university education. Chinese participants were less likely to be overweight $\left(\geq 25 \cdot 0 \mathrm{~kg} / \mathrm{m}^{2}\right.$ ) as compared with the other participants. Overall, the percentage of cigarette smokers reduced from $14.0 \%$ before pregnancy to $2.5 \%$ during pregnancy and the percentage of alcohol consumers reduced from $35.9 \%$ before pregnancy to $2 \cdot 0 \%$ during pregnancy. The percentage of participants who reported going through confinement was lower in Indian (79.6\%) as compared with Chinese (94.8\%) and Malay (91.6\%) participants. Except for ethnicity, characteristics of participants who went through confinement were not significantly different from those of participants who did not.

\section{Dietary changes}

We found that in five out of seventeen foods taken during pregnancy and twenty-one out of thirty-two foods taken during the postpartum period, changes in amount consumed were significantly associated with ethnicity (see Supplementary Tables 1 and 2, respectively, in the online supplementary material). Changes in food consumption are therefore presented separately for each ethnic group, listing the five food items with the greatest percentages of participants in the 'increased' or 'decreased' categories.

Changes in food consumption of the participants during pregnancy as compared with the pre-pregnancy period are 
Table 1 Sociodemographic information of participants: pregnant women ( $n$ 1027) of Chinese, Malay and Indian ethnicity, Growing Up in Singapore Towards healthy Outcomes (GUSTO) study, recruited June 2009 to September 2010

\begin{tabular}{|c|c|c|c|c|c|c|c|c|c|}
\hline \multirow[b]{2}{*}{ Variable } & \multicolumn{2}{|c|}{$\begin{array}{l}\text { Total study population } \\
\qquad(n 1027)^{\star}\end{array}$} & \multicolumn{2}{|c|}{$\begin{array}{l}\text { Chinese } \\
(n 580)\end{array}$} & \multicolumn{2}{|c|}{$\begin{array}{l}\text { Malay } \\
(n 262)\end{array}$} & \multicolumn{2}{|c|}{$\begin{array}{l}\text { Indian } \\
(n \text { 185) }\end{array}$} & \multirow[b]{2}{*}{$P$ valuet } \\
\hline & Mean or $n$ & sd or $\%$ & Mean or $n$ & SD or $\%$ & Mean or $n$ & SD or $\%$ & Mean or $n$ & SD or $\%$ & \\
\hline Age (years) & $30 \cdot 4$ & $5 \cdot 2$ & $31 \cdot 4^{\mathrm{a}}$ & $4 \cdot 9$ & $28 \cdot 5^{\mathrm{b}}$ & $5 \cdot 3$ & $30 \cdot 0^{a, b}$ & $5 \cdot 0$ & $<0.001$ \\
\hline Went through confinement & 817 & $91 \cdot 4$ & 496 & $\underset{a}{94 \cdot 8^{a}}$ & 196 & $91 \cdot 6^{a, b}$ & 125 & $\begin{array}{c}79 \cdot 6^{b} \\
a, c\end{array}$ & $\begin{array}{l}<0.001 \\
<0.001\end{array}$ \\
\hline Primary or less & 43 & $4 \cdot 3$ & 22 & 3.9 & 16 & $6 \cdot 3$ & 5 & $2 \cdot 8$ & \\
\hline Secondary or vocational training & 372 & $37 \cdot 1$ & 160 & $28 \cdot 1$ & 164 & $64 \cdot 1$ & 48 & $27 \cdot 1$ & \\
\hline Pre-university & 250 & $24 \cdot 9$ & 143 & $25 \cdot 1$ & 63 & $24 \cdot 6$ & 44 & $24 \cdot 9$ & \\
\hline University & 338 & $33 \cdot 7$ & 245 & $43 \cdot 0$ & 13 & $5 \cdot 1$ & 80 & $45 \cdot 2$ & \\
\hline Monthly household income (\$S) & & & & a & & $\mathrm{b}$ & & $\mathrm{c}$ & $<0.001$ \\
\hline 0-1999 & 151 & $15 \cdot 8$ & 60 & $11 \cdot 2$ & 62 & $24 \cdot 8$ & 29 & $17 \cdot 2$ & \\
\hline 2000-3999 & 291 & $30 \cdot 5$ & 121 & $22 \cdot 6$ & 111 & $44 \cdot 4$ & 59 & $34 \cdot 9$ & \\
\hline $4000-5999$ & 238 & $25 \cdot 0$ & 140 & $26 \cdot 2$ & 61 & $24 \cdot 4$ & 37 & $21 \cdot 9$ & \\
\hline$\geq 6000$ & 274 & $28 \cdot 7$ & 214 & $40 \cdot 0$ & 16 & $6 \cdot 4$ & 44 & $26 \cdot 0$ & \\
\hline Smoking regularly before pregnancy & 142 & $14 \cdot 0$ & 59 & $10 \cdot 2^{a}$ & 73 & $28 \cdot 3^{\mathrm{b}}$ & 10 & $5 \cdot 4^{\mathrm{a}, \mathrm{c}}$ & $<0.001$ \\
\hline Smoking during pregnancy & 25 & $2 \cdot 5$ & 13 & $2 \cdot 3$ & 11 & $4 \cdot 3$ & 1 & 0.5 & 0.040 \\
\hline Alcohol consumption before pregnancy & 365 & $35 \cdot 9$ & 284 & $49 \cdot 4^{a}$ & 48 & $18 \cdot 5^{\mathrm{b}}$ & 33 & $17 \cdot 9^{\mathrm{b}}$ & $<0.001$ \\
\hline Alcohol consumption during pregnancy & 20 & $2 \cdot 0$ & 18 & $3 \cdot 2$ & 1 & 0.4 & 1 & $0 \cdot 6$ & 0.009 \\
\hline Pre-pregnancy BMl $\left(\mathrm{kg} / \mathrm{m}^{2}\right)$ & & & & a & & & & & $<0.001$ \\
\hline$<18.5$ & 120 & $12 \cdot 7$ & 78 & $14 \cdot 6$ & 26 & $10 \cdot 9$ & 16 & $9 \cdot 3$ & \\
\hline $18 \cdot 5-24 \cdot 9$ & 599 & $63 \cdot 5$ & 379 & $71 \cdot 1$ & 130 & $54 \cdot 6$ & 90 & $52 \cdot 3$ & \\
\hline $25 \cdot 0-29 \cdot 9$ & 159 & $16 \cdot 9$ & 60 & $11 \cdot 3$ & 49 & $20 \cdot 6$ & 50 & $29 \cdot 1$ & \\
\hline$\geq 30 \cdot 0$ & 65 & $6 \cdot 9$ & 16 & $3 \cdot 0$ & 33 & $13 \cdot 9$ & 16 & $9 \cdot 3$ & \\
\hline Weight gain up to $26-28$ weeks' gestation $(\mathrm{kg})$ & $8 \cdot 5$ & $4 \cdot 6$ & $8 \cdot 4$ & $3 \cdot 8$ & $9 \cdot 1$ & $5 \cdot 8$ & $8 \cdot 1$ & $4 \cdot 8$ & 0.069 \\
\hline Daily energy intake at 26-28 weeks' gestation (kJ) & 7803 & 2481 & $8109^{a}$ & 2364 & $7456^{\mathrm{a}, \mathrm{b}}$ & 2778 & $7335^{\mathrm{b}}$ & 2264 & $<0.001$ \\
\hline Daily energy intake at $26-28$ weeks' gestation (kcal) & 1865 & 593 & $1938^{\mathrm{a}}$ & 565 & $1782^{\mathrm{a}, \mathrm{b}}$ & 664 & $1753^{\mathrm{b}}$ & 541 & $<0.001$ \\
\hline Breast-feeding at 3 weeks postpartum & 740 & $82 \cdot 7$ & 441 & $84 \cdot 0$ & 164 & $77 \cdot 0$ & 135 & $86 \cdot 0$ & 0.036 \\
\hline
\end{tabular}

Values are presented as mean and standard deviation for 'age', 'weight gain up to 26-28 weeks' gestation' and 'daily energy intake at 26-28 weeks' gestation'; all other variables as numbers and percentages. Percentages may not add up to $100 \%$ due to rounding.

a,b,c Values within a row with unlike superscript letters were significantly different $(P<0.00033)$

'Number of missing data: $n 11$ for 'age', $n 133$ for 'went through confinement', $n 24$ for 'highest education level', $n 73$ for 'monthly household income', $n 9$ for 'smoking regularly before pregnancy', $n 8$ for 'smoking during pregnancy', $n 9$ for 'alcohol consumption before pregnancy', $n 31$ for 'alcohol consumption during pregnancy', $n 84$ for 'pre-pregnancy BMl', $n 92$ for 'weight gain up to $26-28$ weeks' gestation', $n 20$ for 'daily energy intake at 26-28 weeks' gestation' and $n 132$ for 'breast-feeding at 3 weeks postpartum'.

tTests were meant to assess differences in sociodemographic characteristics across ethnic groups; one-way ANOVA was used for 'age', 'weight gain up to 26-28 weeks' gestation' and 'daily energy intake at 26-28 weeks' gestation' and Pearson's $\chi^{2}$ test of independence was used for other variables. 
Table 2 Foods with substantial increased or decreased consumption during pregnancy as compared with the pre-pregnancy period for each ethnic group (only top five listed): pregnant women ( $n$ 1019) of Chinese, Malay and Indian ethnicity, Growing Up in Singapore Towards healthy Outcomes (GUSTO) study, recruited June 2009 to September 2010

\begin{tabular}{|c|c|c|c|c|c|}
\hline \multicolumn{3}{|c|}{ Foods with increased consumption } & \multicolumn{3}{|c|}{ Foods with decreased consumption } \\
\hline & $\%$ increased & $\%$ decreased & & $\%$ increased & $\%$ decreased \\
\hline Chinese ( $n$ 576) & & & Chinese $(n 576)$ & & \\
\hline Milk & $63 \cdot 7$ & $9 \cdot 7$ & Seafood & $10 \cdot 6$ & $44 \cdot 8$ \\
\hline Fruit & $60 \cdot 8$ & $5 \cdot 7$ & Tea & $3 \cdot 7$ & $41 \cdot 3$ \\
\hline Vegetables & $49 \cdot 1$ & $5 \cdot 4$ & Soft drinks & $11 \cdot 8$ & $41 \cdot 1$ \\
\hline Fish & $44 \cdot 3$ & $17 \cdot 7$ & Coffee & $3 \cdot 0$ & $33 \cdot 3$ \\
\hline Rice, noodles \& bread & $39 \cdot 2$ & $8 \cdot 9$ & Confectionery* & $25 \cdot 7$ & $30 \cdot 6$ \\
\hline Malay (n 259) & & & Malay (n 259) & & \\
\hline Milk & $58 \cdot 7$ & $12 \cdot 7$ & Seafood & $9 \cdot 7$ & $42 \cdot 1$ \\
\hline Fruit & $52 \cdot 9$ & $12 \cdot 7$ & Soft drinks & $17 \cdot 0$ & $40 \cdot 9$ \\
\hline Rice, noodles \& bread & $47 \cdot 1$ & $12 \cdot 4$ & Tea & $17 \cdot 0$ & $33 \cdot 6$ \\
\hline Vegetables & $45 \cdot 6$ & $12 \cdot 4$ & Coffee & $4 \cdot 6$ & $32 \cdot 1$ \\
\hline Fish & $37 \cdot 5$ & $24 \cdot 3$ & Eggs & $12 \cdot 0$ & $28 \cdot 6$ \\
\hline Indian ( $n$ 184) & & & Indian ( $n$ 184) & & \\
\hline Fruit & $66 \cdot 9$ & $11 \cdot 4$ & Soft drinks & $9 \cdot 8$ & $36 \cdot 4$ \\
\hline Milk & $66 \cdot 3$ & $10 \cdot 9$ & Tea & $8 \cdot 2$ & $36 \cdot 4$ \\
\hline Vegetables & $64 \cdot 7$ & $3 \cdot 8$ & Coffee & $6 \cdot 0$ & $35 \cdot 9$ \\
\hline Rice, noodles \& bread & $42 \cdot 4$ & $10 \cdot 3$ & Confectionery & $20 \cdot 1$ & $35 \cdot 3$ \\
\hline Cheese \& yoghurt & $33 \cdot 2$ & $16 \cdot 3$ & Meat & $8 \cdot 1$ & $33 \cdot 7$ \\
\hline
\end{tabular}

Pearson's $\chi^{2}$ test of goodness-of-fit was used to test if the percentages in the 'increased' and 'decreased' categories varied significantly from the expected proportion of $50 \%$ in each category. $P$ values were all $<0.001$ for food items shown in the table except for confectionery $(P=0.120$ and 0.006 for Chinese and Indian, respectively), fish for Malay $(P=0.007)$ and cheese and yoghurt for Indian $(P=0.001)$. Other food items that were assessed but not listed in the table are chicken, organ meats, chocolate drinks and wine/alcohol; for percentages of other columns ('same as before' and 'do not usually eat'), please refer to Supplementary Table 1.

${ }^{\star}$ Confectionery includes chocolates, sweets, biscuits and cakes.

presented in Table 2. For all three ethnic groups, 39.2\% to $66.9 \%$ of the participants increased their consumption of milk, fruit, vegetables, and rice, noodles and bread $(P<0.001$ for all items). More participants also increased rather than decreased their fish intakes. In contrast, for all three ethnic groups, more participants decreased rather than increased their consumption of tea, coffee, soft drinks and seafood $(P<0 \cdot 001$ for all items). Moreover, confectionery consumption was decreased in about a third of all participants. Ethnic differences in dietary changes during pregnancy were modest. The largest ethnic difference observed was that of eggs consumption, which was decreased in $28.6 \%$ of the Malay participants as compared with $11 \cdot 1 \%$ for Chinese and $16.9 \%$ for Indians ( $P$ for ethnic difference $<0 \cdot 001$ ). For other foods, the percentages of participants reporting increased or decreased consumption during pregnancy were smaller (see Supplementary Table 1).

Table 3 shows the changes in food consumption of the participants during the postpartum period as compared with the pre-pregnancy period. In all three ethnic groups, consumption of fish, leafy vegetables and milk-based drinks was increased in a large percentage of the participants (47.1\% to $73 \cdot 4 \% ; P<0 \cdot 001$ for all items). In contrast, chocolates and sweets and noodle consumption during the postpartum period was decreased in approximately half of the participants $(P<0 \cdot 001$ for all items). Furthermore, substantially more participants decreased rather than increased their intakes of seafood, cheese and yoghurt, fruit juice and soft drinks in all three ethnic groups $(P<0.001$ for all items).
Substantial ethnic differences in dietary changes were observed during the postpartum period. A larger proportion of the Chinese participants $(87 \cdot 2 \%)$ increased their ginger consumption, as compared with smaller percentages of Indian $(40 \cdot 8 \%)$ and Malay $(31 \cdot 8 \%)$ participants $(P$ for ethnic difference $<0 \cdot 001)$. Similar ethnic differences were observed for wine/alcohol used in cooking, herbs and spices, and herbal tea consumption (see Supplementary Table 2). In contrast, more than half of the Malay (73.8\%) and Indian (56.7\%) participants increased their plain water consumption, while more than half of the Chinese participants $(53 \cdot 2 \%)$ decreased their water consumption ( $P$ for ethnic difference $<0 \cdot 001$ ). More than half of the Indian participants increased their milk and garlic consumption, as compared with smaller percentages of the Chinese and Malay participants ( $P$ for ethnic difference $<0 \cdot 001$ ). About half of the Malay participants decreased their eggs and beef consumption, as compared with less than a third of the Chinese and Indian participants. For other assessed foods, the percentages of participants reporting increased or decreased consumption during the postpartum period were smaller (see Supplementary Table 2). Restricting the analysis to participants who went through confinement did not change the results substantially.

We also conducted analyses stratified by pre-pregnancy overweight status (BMI $<25 \cdot 0 \mathrm{~kg} / \mathrm{m}^{2}$ or $\geq 25 \cdot 0 \mathrm{~kg} / \mathrm{m}^{2}$ ), weight gain during pregnancy (less than the mean or greater than or equal to the mean) and breast-feeding practice (yes/no). Overweight women were more likely to reduce their intakes of meat and eggs during pregnancy 
Table 3 Foods with substantial increased or decreased consumption during postpartum as compared with the pre-pregnancy period for each ethnic group (only top five listed): pregnant women ( $n$ 895) of Chinese, Malay and Indian ethnicity, Growing Up in Singapore Towards healthy Outcomes (GUSTO) study, recruited June 2009 to September 2010

\begin{tabular}{|c|c|c|c|c|c|}
\hline \multicolumn{3}{|c|}{ Foods with increased consumption } & \multicolumn{3}{|c|}{ Foods with decreased consumption } \\
\hline & $\%$ increased & $\%$ decreased & & $\%$ increased & $\%$ decreased \\
\hline Chinese ( $n$ 524) & & & Chinese ( $n$ 524) & & \\
\hline Ginger & $87 \cdot 2$ & $4 \cdot 8$ & Chocolates \& sweets & $8 \cdot 2$ & $56 \cdot 3$ \\
\hline Wine/alcohol used in cooking & $71 \cdot 8$ & $9 \cdot 0$ & Plain water & $26 \cdot 7$ & $53 \cdot 2$ \\
\hline Herbs \& spices & $70 \cdot 4$ & $9 \cdot 7$ & Fruit juice & $3 \cdot 6$ & $53 \cdot 1$ \\
\hline Fish & $65 \cdot 7$ & $12 \cdot 6$ & Seafood & 1.9 & $50 \cdot 6$ \\
\hline Pork & $57 \cdot 8$ & $11 \cdot 1$ & Cheese \& yoghurt & $4 \cdot 2$ & $46 \cdot 6$ \\
\hline Malay (n 214) & & & Malay $(n 214)$ & & \\
\hline Plain water & $73 \cdot 8$ & $9 \cdot 8$ & Noodles & $7 \cdot 0$ & $53 \cdot 3$ \\
\hline Leafy vegetables & $73 \cdot 4$ & $3 \cdot 7$ & Chicken & $7 \cdot 9$ & $49 \cdot 1$ \\
\hline Milk-based drinks & $58 \cdot 4$ & $14 \cdot 0$ & Chocolates \& sweets & $9 \cdot 8$ & $47 \cdot 7$ \\
\hline Fish & $55 \cdot 6$ & $16 \cdot 4$ & Eggs & $6 \cdot 5$ & $47 \cdot 7$ \\
\hline Non-leafy vegetables & $47 \cdot 7$ & $15 \cdot 9$ & Beef & 8.9 & $46 \cdot 3$ \\
\hline Indian ( $n$ 157) & & & Indian ( $n$ 157) & & \\
\hline Leafy vegetables & $58 \cdot 0$ & $11 \cdot 5$ & Seafood & $2 \cdot 6$ & $44 \cdot 6$ \\
\hline Plain water & $56 \cdot 7$ & $18 \cdot 5$ & Noodles & $2 \cdot 6$ & $43 \cdot 3$ \\
\hline Milk & $55 \cdot 4$ & $13 \cdot 4$ & Chocolates \& sweets & $7 \cdot 6$ & $40 \cdot 8$ \\
\hline Garlic & $53 \cdot 5$ & $6 \cdot 4$ & Soft drinks & 1.9 & $38 \cdot 2$ \\
\hline Fish & $51 \cdot 6$ & $10 \cdot 2$ & Fruit juice & $12 \cdot 7$ & $37 \cdot 6$ \\
\hline
\end{tabular}

Pearson's $\chi^{2}$ test of goodness-of-fit was used to test if the percentages in the 'increased' and 'decreased' categories varied significantly from the expected proportion of $50 \%$ in each category. $P$ values were all $<0.001$ for food items shown in the table. Other food items that were assessed but not listed in the table are mutton, pork trotters, organ meats, nuts, fruit, rice, bread, oil, herbal tea, tea, coffee and wine/alcohol; for percentages of other columns ('same as before' and 'do not usually eat'), please refer to Supplementary Table 2.

and to increase their intakes of garlic and plain water during the postpartum period. In contrast, non-overweight women were more likely to increase their intakes of chicken, eggs and oil in the postpartum period. Women who gained more weight were more likely to decrease their intakes of rice and chocolates and sweets during the postpartum period. Finally, breast-feeding women were more likely to increase their intakes of fish and milk during the postpartum period.

\section{Discussion}

A large majority of the Singaporean women of Chinese, Malay and Indian ethnicity in the present study indicated that they observed the confinement period. Moreover, consumption of many foods changed during pregnancy and the postpartum period. Changes in food consumption during pregnancy were generally similar for Chinese, Malay and Indian women. For example, milk, fruit and vegetables consumption was increased while tea, coffee and soft drinks consumption was decreased. During the postpartum period, many increased their consumption of fish, leafy vegetables and milk-based drinks while substantially more participants decreased rather than increased their consumption of chocolates and sweets, noodles, seafood, cheese and yoghurt, fruit juice and soft drinks. Large ethnic differences in dietary practice were observed during the postpartum period e.g. for increases in ginger, milk and garlic consumption.

The baseline dietary information (pre-pregnancy) of the study participants was not collected. However, according to the National Nutrition Survey ${ }^{(14)}$ in Singapore, on average, women aged 18-39 years meet national dietary recommendations of 5-7 servings of rice and alternatives (e.g. bread, noodles), $\geq 2$ servings of vegetables and 2-3 servings of meat and alternatives (e.g. prawns, milk) daily. However, they do not meet the recommended servings for fruit ( $\geq 2$ servings/d). The survey also indicated dietary differences among the ethnic races. For example, Chinese in Singapore consume more rice, porridge, noodles, red meat and poultry as compared with the Malays and Indians in Singapore.

Physiological changes during pregnancy can impact on dietary needs. Not only is higher energy intake recommended during the second half of the pregnancy, a higher increment in intakes of various nutrients including protein, $\mathrm{Ca}$, folate, vitamin $\mathrm{C}$ and vitamin $\mathrm{D}$ is recommended during pregnancy ${ }^{(15)}$. Increasing the consumption of nutrient-dense fruit and vegetables and lowering the intake of foods with low nutrient density are thus advised during pregnancy ${ }^{(15)}$. It is possible that participants might have increased their fruit and vegetables intakes due to modern medical and nutritional advice which usually promotes fruit and vegetables consumption during pregnancy ${ }^{(16)}$. At the same time, in traditional Asian belief, pregnancy is generally considered to be a 'hot' state and thus women need to consume more 'cold' foods to maintain the 'hot-cold balance' ${ }^{(8)}$. As most vegetables and fruit are considered 'cold' ${ }^{(4,5)}$, it is also possible that a large proportion of the participants from all ethnic groups is following these cultural practices and thus increased their intakes of this food group. The increased milk consumption during pregnancy in all three ethnic groups 
in the study could be due to modern dietary advice that promotes milk products as good sources of $\mathrm{Ca}$ and other nutrients $^{(15)}$. Insufficient Ca intake during pregnancy may lead to lower $\mathrm{Ca}$ content in the fetus' skeleton ${ }^{(15)}$. Tea and coffee intakes were decreased in all three ethnic groups, likely as a consequence of the reported negative effects of caffeine on pregnancy outcomes ${ }^{(17,18)}$.

Similar to pregnancy, mothers also need to meet higher nutritional requirements during the postpartum period ${ }^{(19)}$. This is especially true for breast-feeding women as nutritional factors can influence the quality and quantity of breast milk ${ }^{(19)}$. It is recommended that a lactating mother increases her energy intake by $2092 \mathrm{~kJ} / \mathrm{d}(500 \mathrm{kcal} / \mathrm{d})$ as compared with the pre-pregnancy period and includes an adequate amount of protein in her diet ${ }^{(19)}$. In traditional Asian belief, the body of a woman is believed to be in a state of 'cold' during the postpartum period and thus 'hot' foods are recommended ${ }^{(4)}$. Ginger, wine and herbs are frequently used in preparation of the Chinese confinement diet due to their warming properties ${ }^{(7,20)}$. Fruit juice is generally perceived as 'cold' and seafood is usually regarded as 'poisonous' during this period ${ }^{(7)}$. Thus, it was not surprising that fruit juice and seafood consumption was decreased in more than half of the Chinese participants in the present study. Furthermore, seafood is prone to $\mathrm{Hg}$ contamination that may be harmful to the central nervous system of the fetus or infant ${ }^{(21)}$. For Malays, decreased consumption of beef and eggs in a large percentage of the participants is consistent with the belief that these foods inhibit a woman's general recovery ${ }^{(5)}$. However, a large percentage of the Malay participants in our study increased their consumption of leafy and non-leafy vegetables, which are traditionally proscribed, probably due to the influence of modern medical advice ${ }^{(19)}$. Classification of garlic and fish as 'hot' foods in Indian belief may be the reason for increased consumption of these foods in more than half of the Indian participants in our study ${ }^{(3,8)}$. Milk consumption was also increased as it is prescribed in the Indian confinement $\operatorname{diet}^{(3,8)}$. The decreased intake of fruit juice in a large percentage of our Indian participants may be due to Indian perception that most fruit are 'cold'(3).

We compared our results with those from earlier studies that examined the dietary changes of Chinese, Malay and Indian women during pregnancy and the postpartum period. In a study conducted in Malaysia, the dietary beliefs of 100 Chinese women (mean age 28.7 (SD 5.0) years) during the postpartum period were assessed and they were generally in agreement with our results ${ }^{(7)}$. In that study, the participants were asked about their acceptance ('prohibited', 'not encouraged', 'neutral', 'encouraged' and 'must eat a lot') and perceived properties ('cold', 'hot', 'poison', 'windy' and 'do not know') of a list of foods during the postpartum period. Ginger and white pepper consumption was thought to be 'encouraged' or 'must eat a lot' by more than half of that study's participants. Furthermore, consumption of most seafood other than fish (e.g. prawns and crab) and fruit juice was considered to be 'prohibited' and 'not encouraged' by most of the participants in that study. In contrast with our findings, most fish was perceived to be 'poison' during the postpartum period by more than half of that study's participants and the consumption of fish was thought to be 'prohibited' or 'not encouraged'. However, the consumption of ikan haruan, a type of fish believed to enhance dermal wound healing, was thought to be 'encouraged' or 'must eat a lot' by a quarter of the participants. In a study conducted in China, dietary behaviours of about 2000 women during the postpartum period were assessed using an intervieweradministered questionnaire and an FFQ. In that study, fish and rice wine were frequently consumed by the Chinese participants during the postpartum period ${ }^{(22)}$. Furthermore, $78.8 \%$ of the participants never ate fruit during this period ${ }^{(22)}$. In another study conducted in Malaysia (information gathered through interviews with informants), Malay women indicated that eggs and beef were generally avoided during the postpartum period ${ }^{(5)}$ as was observed in our study. However, plain water and most vegetables were proscribed in that population ${ }^{(5)}$, whereas consumption of these foods was increased during the postpartum period in our study. In general, dietary changes in our study that appear to be based on traditional belief (e.g. regarding ginger, wine used in cooking during the postpartum period) were also observed in earlier studies. However, the increased consumption of fruit, vegetables and fish during the postpartum period indicated that our study population may have been more affected by modern dietary advice than the participants of the earlier studies.

Our study had several strengths. Its sample size was reasonably large with a representation of three major ethnic groups in Asia. In addition, the questionnaires used to assess changes in food consumption were intervieweradministered which may have improved the completeness of the data. However, our study also had several limitations. First, our questionnaire only assessed relative changes of food consumption as compared with the pre-pregnancy period and did not allow estimation of absolute changes in food consumption. Second, participants were recruited only from public hospitals in Singapore and women with the highest incomes may have been under-represented as they may have chosen private hospitals. However, KKH alone delivers approximately $30 \%$ of the babies born in Singapore annually ${ }^{(23)}$. Therefore, the study population can be expected to be reasonably representative of the overall population of pregnant women in Singapore.

Our results may have several implications for dietary intervention during pregnancy and the postpartum period. Because traditional beliefs appear to have important influences on the dietary choices of various Asian groups, dietary intervention targeting Asian pregnant and lactating women should take these traditional beliefs into consideration. For instance, most vegetables and fruit are considered 'cold' and 
are not usually consumed during the postpartum period ${ }^{(7)}$. This not only poses a risk to the mother's health due to the lack of dietary fibre, vitamins, minerals and phytochemicals, but may also influence the infant's acceptance of fruit and vegetables later in life if the mother is breast-feeding ${ }^{(24)}$. Dietary intervention involving an increase in fruit and vegetables consumption during the postpartum period can focus on promoting more acceptable fruit and vegetables such as apple, black fungus and red dates for Chinese ${ }^{(7,25)}$, tamarind and turmeric root (usually used to make juice or concoction) for Malays ${ }^{(5)}$, and ginger, beans and tomato for Indians ${ }^{(3,8)}$. Chicken soup prepared with sesame seed oil and rice wine is commonly consumed by Chinese during the postpartum period for its warming effect ${ }^{(26)}$. However, recent research has shown that the consumption of this alcoholic soup significantly lengthens milk ejection time and lowers milk yield of lactating mothers ${ }^{(26)}$. Furthermore, alcohol can be detected in breast milk for several hours after consumption of the alcoholic soup ${ }^{(27)}$. Results from some studies suggest that alcohol in breast milk can be detrimental for the sleep pattern and psychomotor development of infants ${ }^{(27)}$. Therefore, consumption of traditional foods such as alcoholic soups should be targeted in dietary advice or intervention for Asian lactating women.

\section{Conclusion}

The practice of confinement is still very common in Chinese, Malay and Indian women in Singapore, a high-income country where Western medicine is widely accepted. Furthermore, consumption of many foods was altered during pregnancy and the postpartum period in a large number of Chinese, Malay and Indian women. These changes appear to reflect a mix of traditional beliefs and modern dietary advice. As such, to improve dietary compliance and ultimately the health of women and their children, it is important to assess an individual's traditional beliefs before conducting any dietary intervention involving Asian pregnant and lactating women.

\section{Acknowledgements}

Sources of funding: GUSTO is part of the Translational Clinical Research (TCR) Flagship Programme on Developmental Pathways to Metabolic Disease funded by the National Research Foundation (NRF) and administered by the National Medical Research Council (NMRC), Singapore. Further support is provided by the Singapore Institute for Clinical Sciences, A*STAR, Singapore. K.G. is supported by the National Institute for Health Research through the NIHR Southampton Biomedical Research Centre. Conflicts of interest: K.G. has acted as a consultant to Abbott Nutrition and Nestlé Nutrition, and has received reimbursement for speaking at an Abbott Nutrition Conference on
Pregnancy Nutrition and Later Health Outcomes and at a Nestlé Nutrition Institute Workshop. He is part of an academic consortium that has received research funding from Abbott Nutrition, Nestec and Danone. None of the other authors report any potential conflict of interest. Authors' contributions: L.-W.C. conducted statistical analyses, interpreted the results and wrote the manuscript. Y.L.L., D.F. and W.M.H. designed the dietary questionnaires. Y.S.C., P.G., K.G., K.K., S.-M.S., S.E.S. and K.H.T. conceptualized and obtained funding for the GUSTO study. M.F.F.C. and R.M.v.D. are joint last authors. M.F.F.C. and R.M.v.D. supervised the analyses and contributed to critical interpretation of the results. All authors provided critical revision of the manuscript. Acknowledgements: The authors thank the GUSTO study group, clinic and home visit staff, and all the participants in the GUSTO study. The GUSTO study group includes Dennis Bier, Arijit Biswas, Cai Shirong, Helen Chan, Jerry Chan, Chan Yiong Huak, Cornelia Chee, Audrey Chia, Chiang Wen Chin, Amutha Chinnadurai, Chng Chai Kiat, Chong Shang Chee, Chua Mei Chien, Wayne Cutfield, Mary Daniel, Ding Chun Ming, Anne Ferguson-Smith, Eric Andrew Finkelstein, Marielle Fortier, Anne Goh, Daniel Goh, Joshua J. Gooley, Mark Hanson, Mikael Hartman, Michael Heymann, Joanna Holbrook, Stephen Hsu Chin-Ying, Hazel Inskip, Jeevesh Kapur, Lee Bee Wah, Lee Yung Seng, B.F.P. Leutscher-Broekman, Lim Sok Bee, Loh Seong Feei, Iliana Magiati, Michael Meaney, Susan Morton, Krishnamoorthy N, Cheryl Ngo, Pang Wei Wei, Prathiba Agarwal, Qiu Anqi, Quah Boon Long, Victor S. Rajadurai, Jen Richmond, Anne Rifkin-Graboi, Allan Sheppard, Lynette Pei-Chi Shek, Borys Shuter, Leher Singh, So Wing Chee, Walter Stunkel, Su Lin Lin, Tan Soek Hui, Teoh Oon Hoe, Mya Thway Tint, Terry Yoke Yin Tong, Hugo Van Bever, Sudhakar Venkatesh, Helena Marieke Verkooijen, Inez By Wong, P.C. Wong, Fabian Yap and George S.H. Yeo.

\section{Supplementary material}

To view supplementary material for this article, please visit http://dx.doi.org/10.1017/S1368980013001730

\section{References}

1. Abu-Saad K \& Fraser D (2010) Maternal nutrition and birth outcomes. Epidemiol Rev 32, 5-25.

2. Hayat L, al-Sughayer MA \& Afzal M (1999) Fatty acid composition of human milk in Kuwaiti mothers. Comp Biochem Physiol B Biochem Mol Biol 124, 261-267.

3. Choudhry UK (1996) Traditional practices of women from India pregnancy childbirth, and newborn care. $J$ Obstet Gynecol Neonatal Nurs 26, 533-539.

4. Pillsbury BL (1978) Doing the month confinement and convalescence of Chinese women after childbirth. Soc Sci Med 12, 11-22.

5. Manderson L (1981) Roasting, smoking and dieting in response to birth: Malay confinement in cross-cultural perspective. Soc Sci Med B 15, 509-520. 
6. Kim-Godwin YS (2003) Postpartum beliefs and practices among non-Western cultures. MCN Am J Matern Child Nurs 28, 74-78.

7. Poh BK, Wong YP \& Norimah AK (2005) Postpartum dietary intakes and food taboos among Chinese women attending maternal and child health clinics and maternity hospital, Kuala Lumpur. Malays J Nutr 11, $1-21$.

8. Nag M (1994) Beliefs and practices about food during pregnancy: implications for maternal nutrition. Econ Polit Wkly 29, 2427-2438.

9. Fishman C, Evans R \& Jenks E (1988) Warm bodies, cool milk: conflicts in post partum food choice for Indochinese women in California. Soc Sci Med 26, 1125-1132.

10. Cheung NF (1997) Chinese zuoyuezi (sitting in for the first month of the postnatal period) in Scotland. Midwifery 13, 55-65.

11. Matthey S, Panasetis P \& Barnett B (2002) Adherence to cultural practices following childbirth in migrant Chinese women and relation to postpartum mood. Health Care Women Int 23, 567-575.

12. Chien L, Tai C, Ko Y et al. (2006) Adherence to 'doing-themonth' practices is associated with fewer physical and depressive symptoms among postpartum women in Taiwan. Res Nurs Health 29, 374-383.

13. Chee CYI, Lee DTS, Chong YS et al. (2005) Confinement and other psychosocial factors in perinatal depression: a transcultural study in Singapore. J Affect Disord 89, 157-166.

14. Health Promotion Board (2004) Report of the National Nutrition Survey 2004, Singapore. http://www.hpb.gov. sg/data/hpb.home/files/whp/health_fac/resources/nns\%20 report_final(merged).pdf (accessed December 2012).

15. Ortega RM (2001) Dietary guidelines for pregnant women. Public Health Nutr 4, 1343-1346.
16. Fowles ER (2006) What's a pregnant woman to eat? A review of current USDA dietary guidelines and mypyramid. I Perinat Educ 15, 28-33.

17. Bakker R, Steegers EAP, Obradov A et al. (2010) Maternal caffeine intake from coffee and tea, fetal growth, and the risks of adverse birth outcomes: the Generation R Study. Am J Clin Nutr 91, 1691-1698.

18. CARE Study Group (2008) Maternal caffeine intake during pregnancy and risk of fetal growth restriction: a large prospective observational study. BMJ 337, a2332.

19. Cervera P \& Ngo J (2001) Dietary guidelines for the breastfeeding woman. Public Health Nutr 4, 1357-1362.

20. Raven JH, Chen Q, Tolhurst RJ et al. (2007) Traditional beliefs and practices in the postpartum period in Fujian Province, China: a qualitative study. BMC Pregnancy Childbirth 7, 8.

21. Dorea JG (2004) Mercury and lead during breast-feeding. Br J Nutr 92, 21-40.

22. Liu N, Mao L, Sun X et al. (2006) Postpartum practices of puerperal women and their influencing factors in three regions of Hubei, China. BMC Public Health 6, 274.

23. Tan KH, Agarwal P, Hoon SJ et al. (2010) Integrated perinatal care and beyond. Proc Singapore Healthcare 19, 124-131.

24. Forestell CA \& Mennella JA (2007) Early determinants of fruit and vegetable acceptance. Pediatrics 120, 1247-1254.

25. Holroyd E, Katie FK, Chun LS et al. (2009) Doing the month an exploration of postpartum practices in Chinese women. Health Care Women Int 18, 301-313.

26. Chien Y-C, Huang Y-J, Hsu C-S et al. (2009) Maternal lactation characteristics after consumption of an alcoholic soup during the postpartum 'doing-the-month' ritual. Public Health Nutr 12, 382-388.

27. Chien YC, Liu J-F, Huang Y-J et al. (2005) Alcohol levels in Chinese lactating mothers after consumption of alcoholic diet during postpartum doing-the-month ritual. Alcohol 37, $143-150$. 